\title{
Comparação de dois métodos DRIS para o diagnóstico de deficiências nutricionais do cafeeiro
}

\author{
Fábio Luiz Partelli(1), Henrique Duarte Vieira(1), Pedro Henrique Monnerat ${ }^{(1)}$ e Alexandre Pio Viana ${ }^{(1)}$
}

(1)Universidade Estadual do Norte Fluminense Darcy Ribeiro, Centro de Ciências e Tecnologias Agropecuárias, CEP 28013-602 Campos dos Goytacazes, RJ. E-mail: partelli@yahoo.com.br, henrique@uenf.br, monnerat@uenf.br, pirapora@uenf.br

\begin{abstract}
Resumo - O objetivo deste trabalho foi comparar as diagnoses nutricionais realizadas pelo DRIS e pelo DRIS modificado (com inclusão da matéria seca), utilizando-se quatro critérios: todas as relações entre nutrientes; as relações com maior razão de variância; as com menor coeficiente de variação e as com valor de relação maior do que um. Foram usados os teores foliares de N, P, K, Ca, Mg, S, B, Cu, Fe, Mn e Zn e a produtividade de 56 lavouras orgânicas de café ‘Conilon’, do Estado do Espírito Santo. Verificou-se, para os diferentes métodos e critérios, a freqüência do nutriente cujo índice foi o mais negativo e o mais positivo, a correlação entre o teor do nutriente e seu índice e a correlação entre produtividade e índice de balanço nutricional. O DRIS e o DRIS modificado (DRIS-M) proporcionaram diagnósticos próximos, e os quatro critérios de cálculo de índices DRIS alteraram a ordem de limitação de nutrientes no cafeeiro. O DRIS-M apresenta correlação um pouco mais elevada entre a produtividade e o índice de balanço nutricional, bem como entre o teor do nutriente e o seu índice DRIS.
\end{abstract}

Termos para indexação: Coffea canephora, procedimentos de cálculos, nutrição mineral.

\section{Comparison of two DRIS methods for diagnosing nutrients deficiencies in coffee trees}

\begin{abstract}
The objective of this work was to compare the nutritional diagnoses by DRIS and DRIS with the incorporation of dry matter (modified DRIS), under four criteria: all ratios between nutrients, nutrient ratios with larger variance ratio, smaller coefficient of variation, and nutrient ratios with values greater than one. Leaf concentrations of N, P, K, Ca, Mg, S, B, Cu, Fe, Mn and Zn, and the productivity of 56 organic crops of 'Conilon' coffee of the State of Espírito Santo, Brazil, were used. The frequency of the nutrient whose index was the most negative and the most positive, the correlation between the nutrient and its index, and the correlation between productivity and nutrient balance index were evaluated. DRIS and DRIS modified (DRIS-M) had provided similar diagnoses, and the four criteria of DRIS indices calculation changed the order of nutrients limitation in coffee trees. DRIS-M presents a little higher correlation between the productivity and the nutrient balance index, as well as, between nutrient concentration and its DRIS index.
\end{abstract}

Index terms: Coffea canephora, procedures of calculations, mineral nutrition.

\section{Introdução}

A diagnose foliar é muito eficiente, pois permite diagnóstico nutricional direto e preciso, já que a planta é considerada o próprio extrator de nutrientes do solo (Beaufils, 1973). A correta interpretação de resultados de análises foliares gera informações que auxiliam no uso racional de insumos, no equilíbrio nutricional das plantas e, conseqüentemente, no aumento da produtividade. Portanto, preconiza-se a utilização de métodos que favoreçam subsídios para um diagnóstico nutricional eficiente e prático, a partir de resultados analíticos das folhas de uma planta ou lavoura.
O Sistema Integrado de Diagnose e Recomendação (DRIS) (Beaufils, 1973) incorpora o conceito de balanço nutricional, ou de equilíbrio entre os minerais nos tecidos das plantas (Baldock \& Schulte, 1996; Lucena, 1997). Esta técnica baseia-se no cálculo de índices DRIS, para cada nutriente, avaliados pela relação entre as razões dos teores de cada elemento com os demais, obtidas de uma amostra com relações padrões, cuja composição mineral é proveniente de uma população de plantas altamente produtivas.

O DRIS é eficiente no diagnóstico nutricional das plantas, pois propicia a determinação da seqüência de limitação nutricional. Índices DRIS positivo e negativo indi- 
cam, respectivamente, excesso ou deficiência de nutriente na planta. Desta forma, quanto mais próximo de zero estiver o índice, mais próximo do equilíbrio estará o elemento (Baldock \& Schulte, 1996).

No diagnóstico nutricional com incorporação dos índices de matéria seca (DRIS-M), além das relações entre os nutrientes, usa-se a concentração deles, comparando-os com as normas (Hallmark et al., 1987a, 1987b). Esse método visa a neutralizar algumas limitações do DRIS, pela incorporação de uma medida real de suficiência do elemento no cálculo do índice, para diminuir a possibilidade de diagnóstico errôneo, nos casos em que há maior concentração ou diluição e as relações dos nutrientes prevalecem constantes. Entretanto, o DRIS-M reintroduz o efeito da idade do tecido foliar sobre a concentração do elemento no processo de diagnóstico, assim, a coleta deve ser padronizada.

Beaufils (1973) sugere que sejam escolhidas, como valores de referência, as relações de nutrientes que apresentem menor variância na população de alta produtividade, em comparação com a de baixa produtividade, ou simplesmente as que apresentem maior razão entre as variâncias das populações de baixa e alta produtividade. Esse procedimento foi adotado por Bailey et al. (1997), Hartz et al. (1998), Nick (1998), Reis Júnior et al. (2002) e Reis Júnior \& Monnerat (2003a); no entanto, podem ser utilizadas todas as relações de nutrientes (Wadt et al., 1999), e também há outras sugestões de critérios, na escolha das relações, que ainda carecem de informações, como o uso da expressão em que o valor da relação seja maior que um (Santos et al., 2004), e uso da relação que apresente menor coeficiente de variação.

O objetivo deste trabalho foi comparar as diagnoses nutricionais pelos métodos DRIS e DRIS-M, sob quatro critérios de seleção das relações de nutrientes a serem utilizados no diagnóstico: todas as relações entre nutrientes; relações com maior razão de variância; relações com menor coeficiente de variação; e relação maior que um.

\section{Material e Métodos}

A coleta das folhas foi efetuada em lavouras de café 'Conilon', com idade entre 5 e 15 anos, cultivadas de forma orgânica, localizadas no Norte do Estado do Espírito Santo, onde predomina clima tropical quente e úmido no verão, com inverno seco, precipitação pluvial anual média de $1.200 \mathrm{~mm}$, concentrada entre os meses de novembro e janeiro. A temperatura média anual é de $23^{\circ} \mathrm{C}$, e as médias máxima e mínima de 29 e $18^{\circ} \mathrm{C}$, respectivamente. O solo predominante é o Latossolo Vermelho-Amarelo distrófico (Embrapa, 1999), com fertilidade de média a baixa e pH em torno de 5 (Agência Nacional de Águas, 2004).

Foram selecionadas 56 lavouras, das quais foram coletadas aproximadamente 150 folhas, em 50 plantas por lavoura, distribuídas aleatoriamente. As folhas coletadas estavam situadas no terceiro e quarto par de folhas do ramo plagiotrópico, a partir do ápice do ramo, localizado no terço mediano superior das plantas (Fullin \& Dadalto, 2001). A coleta foi realizada em dezembro de 2003 e janeiro de 2004.

Foi criado um banco de dados, para separar os dados das lavouras de alta e baixa produtividade, com informações sobre os teores dos nutrientes ( $\mathrm{N}, \mathrm{P}, \mathrm{K}, \mathrm{Ca}, \mathrm{Mg}, \mathrm{S}, \mathrm{B}$, $\mathrm{Cu}, \mathrm{Fe}, \mathrm{Mn}$ e Zn) quantificados segundo Silva (1999), e os dados de produtividade de cada lavoura. Os macronutrientes e micronutrientes foram avaliados em $\mathrm{g} \mathrm{kg}^{-1}$ e mg kg${ }^{-1}$ de matéria seca, respectivamente. Foram consideradas as lavouras de alta produtividade (igual ou superior a 40 sacas de café beneficiadas), e as de baixa produtividade (menos de 40 sacas de $60 \mathrm{~kg} \mathrm{ha}^{-1}$ ).

A norma DRIS (média e desvio-padrão), utilizada na realização do diagnóstico, foi proveniente de 22 lavouras, entre as 56 , que apresentaram alta produtividade e teores de nutrientes com distribuição normal.

Os cálculos dos índices dos nutrientes foram realizados por meio do DRIS (apenas com as relações entre nutrientes) e pelo DRIS-M (com o teor do nutriente e as relações entre nutrientes) (Hallmark et al., 1987a), utilizando-se todas as relações entre nutrientes, relações com maior razão de variância, relações com menor coeficiente de variação e valor da relação maior que um.

Para avaliar as oito diferentes formas dos cálculos dos índices, foi quantificado o número de vezes em que os nutrientes se apresentaram mais negativos e mais positivos, como é feito no DRIS. As freqüências observadas nos dois diferentes métodos e nos diferentes critérios (tipos de diagnósticos) foram avaliadas por meio do teste de qui-quadrado, conforme utilizado por Wadt et al. (1998b) a 5\% de probabilidade. Correlacionou-se o teor do nutriente com o seu respectivo índice, assim como a produtividade com o índice de balanço nutricional (IBN) a $1 \%$ de probabilidade.

No cálculo da relação normal reduzida dos teores de dois nutrientes, utilizou-se o método de Jones (1981), que é uma modificação da fórmula original de Beaufils (1973) apresentada na forma estudentizada. Utilizou-se esse método em decorrência da sua facilidade de ope- 
ração (Alvarez Venegas \& Leite, 1999), por fornecer bom diagnóstico (Nick, 1998; Wadt et al., 1998a; Alvarez Venegas \& Leite, 1999), por apresentar diagnósticos semelhantes aos outros métodos (Wadt et al., 1998b) e, segundo Mourão Filho et al. (2002), por ser superior aos métodos de Beaufils (1973).

O método de Jones (1981) baseia-se na seguinte equação: $f(Y / X)=(Y / X a-Y / X n) k / s$, em que, $f(Y / X)$ é a função calculada da relação de nutrientes $\mathrm{Y}$ e $\mathrm{X}$; Y/Xa é a relação de nutrientes da amostra a ser diagnosticada; Y/Xn é a relação de nutrientes da norma; s é o desvio-padrão da relação Y/Xn; k é a constante de sensibilidade que, neste trabalho, foi de 10.

O índice DRIS de um nutriente representa a média aritmética dos quocientes do teor desse nutriente com os teores dos demais nutrientes. Neste trabalho, foram utilizadas as relações diretas e inversas, sugeridas por Alvarez Venegas \& Leite (1999), pois, a função $(\mathrm{f}=\mathrm{N} / \mathrm{P})$ positiva representa alto $\mathrm{N}$, comparado com $\mathrm{P}$, e baixo $\mathrm{P}$ relativo a $\mathrm{N}$, o que contribui de forma positiva para o índice $\mathrm{N}$ e de forma negativa para o índice P. Assim, o índice DRIS foi calculado pela equação:

Índice $X=\left\{\left[f\left(X / Y_{1}\right)+f\left(X / Y_{2}\right)+\ldots+f\left(X / Y_{n}\right)\right]-\left[f\left(Z_{1} / X\right)\right.\right.$ $\left.\left.+\mathrm{f}\left(\mathrm{Z}_{2} / \mathrm{X}\right)+\ldots+\mathrm{f}\left(\mathrm{Z}_{\mathrm{m}} / \mathrm{X}\right)\right]\right\} /(\mathrm{n}+\mathrm{m})$, em que, $\mathrm{Y}_{1}, \mathrm{Y}_{2}, \ldots \mathrm{Y}_{\mathrm{n}}$ são os nutrientes que aparecem no denominador das relações com o nutriente $\mathrm{X} ; \mathrm{Z}_{1}, \mathrm{Z}_{2}, \ldots \mathrm{Z}_{\mathrm{m}}$ são os nutrientes que aparecem no numerador das relações com o nutriente $\mathrm{X}$; $\mathrm{n}$ é o número de funções onde o nutriente $\mathrm{X}$ aparece no numerador; $\mathrm{m}$ é o número de funções onde o nutriente $\mathrm{X}$ aparece no denominador.

O IBN consiste no somatório, em módulo, de todos os índices envolvidos (Sumner, 1977) e foi obtido pela equação: $\mathrm{IBN}=\mid$ índice $\mathrm{x}|+|$ índice $\mathrm{x}|+\ldots+|$ índice $\mathrm{n} \mid$ em que, índice $x$, índice x’ e índice n, são os índices de cada nutriente envolvido no diagnóstico.

\section{Resultados e Discussão}

Pelo teste qui-quadrado, não se observou diferença entre os métodos DRIS e DRIS-M, como relatado por Nick (1998), e entre os quatro critérios estudados. Comparando-se os oito procedimentos de diagnóstico nutricional entre si, nas lavouras orgânicas, apenas o Mg não apresentou diferença em sua quantificação, seja como nutriente com índice mais negativo, seja como o mais positivo (Tabela 1). Esta igualdade de ocorrência no diagnóstico também ocorreu para o $\mathrm{N}$ e para o $\mathrm{B}$, como o nutriente com índice mais positivo e mais negativo, respectivamente.

Tabela 1. Freqüência de índices de nutrientes em que as ordens de limitação se apresentaram com índices mais negativos (+N) e mais positivos (+P), nas 56 lavouras de café orgânico, considerando-se os métodos DRIS e DRIS-M e quatro critérios ${ }^{(1)}$.

\begin{tabular}{|c|c|c|c|c|c|c|c|c|c|}
\hline \multirow[t]{2}{*}{ Nutriente } & \multirow[t]{2}{*}{ Ordem de limitação } & \multicolumn{4}{|c|}{ DRIS-M } & \multicolumn{4}{|c|}{ DRIS } \\
\hline & & TR & $\mathrm{R}>1$ & $\mathrm{R}<\mathrm{CV}$ & $\mathrm{R}>\mathrm{RV}$ & TR & $\mathrm{R}>1$ & $\mathrm{R}<\mathrm{CV}$ & $\mathrm{R}>\mathrm{RV}$ \\
\hline \multirow[t]{2}{*}{$\mathrm{N}$} & $+\mathrm{N}$ & 7 & 11 & 11 & 10 & 7 & 11 & 14 & 11 \\
\hline & $+\mathrm{P}$ & 0 & 0 & 0 & 0 & 0 & 0 & 0 & 0 \\
\hline \multirow[t]{2}{*}{$\mathrm{P}$} & $+\mathrm{N}$ & 6 & 11 & 7 & 7 & 5 & 8 & 6 & 5 \\
\hline & $+\mathrm{P}$ & 7 & 7 & 7 & 7 & 7 & 7 & 7 & 6 \\
\hline \multirow[t]{2}{*}{$\mathrm{K}$} & $+\mathrm{N}$ & 3 & 1 & 2 & 2 & 3 & 1 & 1 & 2 \\
\hline & $+\mathrm{P}$ & 13 & 13 & 15 & 13 & 13 & 13 & 13 & 11 \\
\hline \multirow[t]{2}{*}{$\mathrm{Ca}$} & $+\mathrm{N}$ & 1 & 0 & 0 & 0 & 1 & 0 & 0 & 1 \\
\hline & $+\mathrm{P}$ & 0 & 1 & 1 & 1 & 0 & 1 & 1 & 1 \\
\hline \multirow[t]{2}{*}{$\mathrm{Mg}$} & $+\mathrm{N}$ & 3 & 3 & 3 & 3 & 3 & 3 & 3 & 3 \\
\hline & $+\mathrm{P}$ & 4 & 4 & 4 & 4 & 4 & 4 & 4 & 4 \\
\hline \multirow[t]{2}{*}{$\mathrm{S}$} & $+\mathrm{N}$ & 0 & 0 & 0 & 0 & 0 & 0 & 0 & 1 \\
\hline & $+\mathrm{P}$ & 1 & 1 & 1 & 2 & 1 & 1 & 1 & 1 \\
\hline \multirow[t]{2}{*}{ B } & $+\mathrm{N}$ & 2 & 2 & 2 & 2 & 2 & 2 & 2 & 2 \\
\hline & $+\mathrm{P}$ & 8 & 8 & 6 & 9 & 8 & 8 & 8 & 10 \\
\hline \multirow[t]{2}{*}{$\mathrm{Cu}$} & $+\mathrm{N}$ & 10 & 8 & 9 & 9 & 10 & 9 & 9 & 10 \\
\hline & $+\mathrm{P}$ & 7 & 7 & 6 & 6 & 7 & 7 & 7 & 7 \\
\hline \multirow[t]{2}{*}{$\mathrm{Fe}$} & $+\mathrm{N}$ & 4 & 4 & 5 & 8 & 4 & 3 & 5 & 6 \\
\hline & $+\mathrm{P}$ & 5 & 4 & 4 & 4 & 5 & 4 & 4 & 4 \\
\hline \multirow[t]{2}{*}{$\mathrm{Mn}$} & $+\mathrm{N}$ & 17 & 14 & 15 & 14 & 18 & 17 & 15 & 14 \\
\hline & $+\mathrm{P}$ & 3 & 3 & 3 & 1 & 3 & 3 & 3 & 3 \\
\hline \multirow[t]{2}{*}{$\mathrm{Zn}$} & $+\mathrm{N}$ & 3 & 2 & 2 & 1 & 3 & 2 & 1 & 1 \\
\hline & $+\mathrm{P}$ & 8 & 8 & 9 & 9 & 8 & 8 & 8 & 9 \\
\hline
\end{tabular}

(1)TR: uso de todas as relações entre nutrientes; $\mathrm{R}>1$ : relações em que o valor é maior que 1 ; $\mathrm{R}<\mathrm{CV}$ : relações que apresentam menor coeficiente de variação; R>RV: relações que apresentam maior razão de variância. 
O diagnóstico nutricional foi próximo entre os oito procedimentos, em relação à quantificação do nutriente que se apresentou com índice mais positivo e negativo. Porém, ao se fazer um ordenamento de nutrientes que se apresentaram mais vezes como limitantes, a ordem de limitação muda para alguns nutrientes, como por exemplo, com uso do DRIS-M, com todas as relações de nutrientes em que os nutrientes mais limitantes foram o $\mathrm{Mn}$, Cu e N; entretanto, ao se utilizar o mesmo método e a metade das relações, com o critério maior que um, o Mn, $\mathrm{N}$ = P e Cu foram os mais limitantes. Ao se utilizar os outros dois critérios, os nutrientes mais limitantes foram o Mn, $\mathrm{N}$ e Cu (Tabela 1). Diferenças de ordenamento também ocorrem entre os diferentes critérios, ao se utilizar o DRIS. Essas diferenças entre os procedimentos são maiores que as apresentadas (Tabela 1), pois, houve diagnóstico em que determinado índice de nutriente passou a ser mais negativo em uma lavoura e deixou de sê-lo em outra, encobertando a diferença entre os procedimentos, apesar de a variação numérica dos índices ser pequena. Portanto, esta situação é indesejável, pois o diagnóstico do estado nutricional da lavoura não deve depender do tipo de procedimento adotado.

Ao se comparar o critério que utiliza todas as relações de nutrientes, com o que usa a metade das relações, em função do critério da razão entre variâncias, nota-se que no primeiro ocorre menor freqüência de $\mathrm{N}$ e maior freqüência de $\mathrm{P}$ como nutrientes mais limitantes, fato também relatado por Wadt et al. (1998b) com eucalipto, ao utilizar o DRIS. Fato semelhante também ocorreu com os outros critérios de escolha de relações de nutrientes, ao se comparar com o uso de todas as relações e com outros nutrientes (Tabela 1).

O uso da relação que apresentou maior razão de variância não afetou de forma significativa o diagnóstico (Tabela 1), como já foi relatado por Wadt et al. (1998b), ao trabalhar com eucalipto.

Houve correlação linear entre os teores dos nutrientes e seus índices $(\mathrm{p}<0,01)$, o que permite sugerir que tais índices podem ser utilizados no diagnóstico nutricional (Tabela 2). As correlações entre nutrientes e respectivos índices, de maneira generalizada, foram maiores para os micronutrientes e menores para $\mathrm{N}$ e Ca.

Ocorreram pequenas diferenças entre a correlação de nutrientes e seus respectivos índices; entre alguns procedimentos, entretanto, os valores médios de correlação apresentaram-se mais elevados quando se utilizou o DRIS-M (Tabela 2), possivelmente, por causa da incorporação do efeito de concentração do nutriente.

Ao se verificar a eficiência do diagnóstico, com o uso das relações com maior razão de variância, conforme Beaufils (1973), Bailey et al. (1997), Hartz et al. (1998), Nick (1998), Wadt et al. (1999) e Reis Júnior \& Monnerat (2003), constatou-se que este critério apresentou valores menores de correlação entre o teor do nutriente e seu índice, em relação aos demais critérios (Tabela 2), inclusive ao que seleciona, para diagnóstico, relações com valores maiores do que um, ignorando, assim, a variabilidade amostral. Por esse resultado, no entanto, demonstra-se que não há necessidade de se ter lavou-

Tabela 2. Valores de correlação (r) entre o teor de nutriente e seu respectivo índice, correlação entre produtividade e índice balanço nutricional (PxIBN), e média do IBN das 56 lavouras de café orgânico, considerando-se os métodos DRIS, DRIS-M e quatro critérios ${ }^{(1)}$.

\begin{tabular}{|c|c|c|c|c|c|c|c|c|}
\hline \multirow[t]{2}{*}{ Nutriente e índice } & \multicolumn{4}{|c|}{ DRIS-M } & \multicolumn{4}{|c|}{ DRIS } \\
\hline & TR & $\mathrm{R}>1$ & $\mathrm{R}<\mathrm{CV}$ & R RV & TR & $\mathrm{R}>1$ & $\mathrm{R}<\mathrm{CV}$ & $\mathrm{R}>\mathrm{RV}$ \\
\hline $\mathrm{N} \times \mathrm{IN}$ & 0,57 & 0,71 & 0,68 & 0,58 & 0,51 & 0,59 & 0,53 & 0,46 \\
\hline $\mathrm{P} \times \mathrm{IP}$ & 0,91 & 0,81 & 0,95 & 0,90 & 0,89 & 0,85 & 0,92 & 0,86 \\
\hline $\mathrm{K} \times \mathrm{IK}$ & 0,86 & 0,89 & 0,87 & 0,87 & 0,85 & 0,86 & 0,84 & 0,84 \\
\hline $\mathrm{Ca} \times \mathrm{ICa}$ & 0,73 & 0,75 & 0,67 & 0,68 & 0,67 & 0,67 & 0,54 & 0,55 \\
\hline $\mathrm{Mg} \times \mathrm{IMg}$ & 0,84 & 0,83 & 0,83 & 0,83 & 0,83 & 0,79 & 0,80 & 0,79 \\
\hline $\mathrm{S} \times \mathrm{IS}$ & 0,80 & 0,78 & 0,82 & 0,80 & 0,77 & 0,72 & 0,76 & 0,73 \\
\hline B $x$ IB & 0,79 & 0,84 & 0,81 & 0,77 & 0,76 & 0,81 & 0,78 & 0,73 \\
\hline $\mathrm{Cu} \times \mathrm{ICu}$ & 0,39 & 0,94 & 0,94 & 0,98 & 0,93 & 0,93 & 0,93 & 0,98 \\
\hline $\mathrm{Fe} \times \mathrm{IFe}$ & 0,95 & 0,97 & 0,96 & 0,89 & 0,95 & 0,95 & 0,95 & 0,84 \\
\hline Mn x IMn & 0,92 & 0,99 & 0,99 & 0,82 & 0,91 & 0,98 & 0,98 & 0,80 \\
\hline $\mathrm{Zn} \times \mathrm{IZn}$ & 0,95 & 0,94 & 0,95 & 0,97 & 0,93 & 0,91 & 0,94 & 0,96 \\
\hline Média IBN & 88,5 & 89,8 & 90,6 & 98,9 & 82,4 & 82,9 & 83,8 & 93,0 \\
\hline $\mathrm{P} \times \mathrm{IBN}$ & $-0,376$ & $-0,368$ & $-0,365$ & $-0,344$ & $-0,352$ & $-0,330$ & $-0,327$ & $-0,316$ \\
\hline
\end{tabular}

(1)TR: uso de todas as relações entre nutrientes; $\mathrm{R}>1$ : relações em que o valor é maior que 1 ; $\mathrm{R}<\mathrm{CV}$ : relações que apresentam menor coeficiente de variação; R>RV: relações que apresentam maior razão de variância; todas as correlações foram significativas a $1 \%$ de probabilidade. 
ras de baixa produtividade, além das de alta produtividade, para se estabelecer normas DRIS.

Maiores valores de IBN ocorreram ao se utilizar a relação cuja razão de variância foi maior, pois os índices de nutrientes apresentaram maiores valores (Tabela 2). Esses resultados corroboram os de Wadt et al. (1999), que realizaram diagnóstico em cafeeiro ‘Conilon' e utilizaram todas as relações de nutrientes e as relações definidas pelo teste F. No entanto, esses autores afirmaram que não foi possível concluir, pelo teste qui-quadrado, qual foi o melhor critério, mas citam que a maior amplitude de valores do IBN pode diagnosticar o desequilíbrio nutricional mais facilmente. Entretanto, determinar se um nutriente é potencialmente limitante ou excessivo, depende do critério adotado que, segundo Wadt et al. (1998b), pode ser um índice, em valor absoluto, maior que o IBN médio ou outros critérios.

A definição da fórmula mais adequada também pode ser estabelecida por meio da correlação entre produtividade e o IBN da lavoura (Mourão Filho et al., 2002). Neste trabalho, a melhor correlação entre a produtividade e o IBN ocorreu quando se utilizou o DRIS-M (com inclusão da matéria seca), quando foram utilizadas todas as relações de nutrientes, seguidas do uso da relação maior que um e menor coeficiente de variação, tendo ficado o procedimento em que se adotou o critério de maior razão de variância com uma correlação menor.

\section{Conclusões}

1. O DRIS e o DRIS-M proporcionam diagnósticos próximos, e os quatro critérios de cálculo de índices DRIS mudam a ordem de limitação de nutrientes no cafeeiro.

2. O DRIS-M apresenta correlação um pouco mais elevada do que o DRIS entre a produtividade e IBN, assim como entre o nutriente e seu respectivo índice.

\section{Agradecimentos}

À Universidade Estadual do Norte Fluminense Darcy Ribeiro, pela bolsa concedida e autorização de uso da estrutura de laboratório; à Faperj e Mibasa, pelo financiamento do trabalho; ao Incaper e Biosystems, pelo suporte na coleta de dados; aos agricultores por disponibilizar as lavouras para efetuar as coletas.

\section{Referências}

AGÊNCIA NACIONAL DE ÁGUAS - ANA. A bacia do Rio Doce: características da bacia. Disponível em: <http://www.ana.gov.br/ cbhriodoce/bacia/caracterizacao.asp\#clima>. Acesso em: 6 out. 2004.

ALVAREZ VENEGAS, V.H.; LEITE, R.A. Fundamentos estatísticos das fórmulas usadas para cálculo dos índices DRIS. Boletim Informativo da Sociedade Brasileira de Ciência do Solo, v.24, p.20-25, 1999.

BAILEY, J.S.; BEATTIE, J.A.M.; KILPATRICK, D.J. The diagnosis and recommendation integrated system (DRIS) for diagnosing the nutrient status of grassland swards. I. Model establishment. Plant and Soil, v.197, p.127-135, 1997.

BALDOCK, J.O.; SCHULTE, E.E. Plant analysis with standardized scores combines DRIS and sufficiency range approaches for corn. Agronomy Journal, v.88, p.448-456, 1996.

BEAUFILS, E.R. Diagnosis and recommendation integrated system (DRIS): a general scheme of experimentation and calibration based on principles developed from research in plant nutrition. Pietermaritzburg, South Africa: University of Natal, 1973. 132p. (Soil science bulletin, 1).

EMBRAPA. Centro Nacional de Pesquisa de Solos (Rio de Janeiro, RJ). Sistema brasileiro de classificação de solos. Rio de Janeiro: Embrapa-CNPS; Brasília: Embrapa-SPI, 1999. 412p.

FULLIN, E.A.; DADALTO, G.G. Avaliação da fertilidade do solo e do estado nutricional das plantas. In: DADALTO, G.G.; FULLIN, E.A. (Ed.). Manual de recomendação de calagem e adubação para o Estado do Espírito Santo: quarta aproximação. Vitória: Seea; Incaper, 2001. p.21-55.

HALLMARK, W.B.; MOOY, C.J. de; PESEK, J. Comparison of two DRIS methods for diagnosing nutrient deficiencies. Journal of Fertilizer Issues, v.4, p.151-158, 1987a.

HALLMARK, W.B.; WALWORTH, J.L.; SUMNER, M.E.; MOOY, C.J. de; PESEK, J.; SHAO, K.P. Separating limiting from non-limiting nutrients. Journal of Plant Nutrition, v.10, p.1381-1390, 1987b.

HARTZ, T.K.; MIYAO, E.M.; VALENCIA, J.G. DRIS evaluation of the nutritional status of processing tomato. HortScience, v.33, p.830-832, 1998.

JONES, C.A. Proposed modifications of the diagnosis and recommendation integrated system (DRIS) for interpreting plant analyses. Communications in Soil Science and Plant Analysis, v.12, p.785-794, 1981.

LUCENA, J.J. Methods of diagnosis of mineral nutrition of plants: a critical review. Acta Horticulturae, v.448, p.179-192, 1997.

MOURÃO FILHO, F. de A.A.; AZEVEDO, J.C.; NICK, J.A. Funções e ordem da razão dos nutrientes no estabelecimento de normas DRIS em laranjeira 'Valência'. Pesquisa Agropecuária Brasileira, v.37, p.185-192, 2002.

NICK, J.A. DRIS para cafeeiros podados. 1998. 87p. Dissertação (Mestrado) - Escola Superior de Agricultura Luiz de Queiroz, Piracicaba. 
REIS JÚNIOR, R. dos A.; CORRÊA, J.B.; CARVALHO, J.G.; GUIMARÃES, P.T.G. Diagnose nutricional de cafeeiros da região sul de Minas Gerais: normas DRIS e teores foliares adequados. Revista Brasileira de Ciência do Solo, v.26, p.801-808, 2002.

REIS JÚNIOR, R. dos A.; MONNERAT, P.H. Norms establishment of the Diagnosis and Recommendation Integrated System (DRIS) for nutritional diagnosis of sugarcane. Pesquisa Agropecuária Brasileira, v.38, p.277-282, 2003a.

SANTOS, A.L. dos; MONNERAT, P.H.; CARVALHO, A.J.C. de. Estabelecimento de normas DRIS para o diagnóstico nutricional do coqueiro-anão verde na região Norte Fluminense. Revista Brasileira de Fruticultura, v.26, p.330-334, 2004.

SILVA, F.C. da (Org.). Manual de análises químicas de solos, plantas e fertilizantes. Brasília: Embrapa-SCT, 1999. 370p.
SUMNER, M.E. Use of DRIS system in foliar diagnosis of crops at high yield levels. Communication in Soil Science and Plant Analysis, v.8, p.251-268, 1977.

WADT, P.G.S.; NOVAIS, R.F. de; ALVAREZ VENEGAS, V.H.; BRAGANÇA, S.M. Alternativas de aplicação do "DRIS" à cultura de café conilon (Coffea canephora Pierre). Scientia Agricola, v.56, p.83-92, 1999.

WADT, P.G.S.; NOVAIS, R.F. de; ALVAREZ VENEGAS, V.H.; FONSECA, S.; BARROS, N.F. Valores de referência para macronutrientes em eucalipto obtidos pelos métodos DRIS e chance matemática. Revista Brasileira de Ciência do Solo, v.22, p.685692, 1998a.

WADT, P.G.S.; NOVAIS, R.F. de; ALVAREZ VENEGAS, V.H.; FONSECA, S.; BARROS, N.F.; DIAS, L.E. Três métodos de cálculo do DRIS para avaliar o potencial de resposta à adubação de árvores de eucalipto. Revista Brasileira de Ciência do Solo, v.22, p.661-666, 1998b.

Recebido em 15 de outubro de 2004 e aprovado em 5 de julho de 2005 